\title{
Intraspecific variation in response to spore-to-spore cultivation in the myxomycete, Didymium squamulosum
}

\section{Winsett, KE}

Department of Biological Sciences, Science and Engineering 632, University of Arkansas, Arkansas, USA

Winsett, KE 2011 - Intraspecific variation in response to spore-to-spore cultivation in the myxomycete, Didymium squamulosum. Mycosphere 2 (5), 555-564, Doi 10.5943/mycosphere/2/2/5

Spore-to-spore cultures were attempted for 94 specimens of Didymium squamulosum using common methods of laboratory cultivation. Specimens varied in age, morphology, method of collection, geographic origin and substratum. Spores from 35 specimens germinated in culture, only nine of which successfully went from spore-to-spore in laboratory culture. No patterns were obvious regarding germination success, plasmodium production or fruiting body formation from the varying factors among the specimens, although no spores from any specimen older than six years germinated.

Key words - laboratory cultivation - myxogastrid - phenotypic plasticity - slime mold

\section{Article information}

Received 10 October 2011

Accepted 20 October 2011

Published online 29 October 2011

Corresponding author: Katherine E. Winsett - e-mail - katherine.winsett@ gmail.com

\section{Introduction}

Since the description of spore-to-spore culture of Didymium difforme and Badhamia utricularis (Berk.) by Lister (1901), an effort has been made to do the same for as many species of myxomycetes (Myxogastrids or plasmodial slime molds) as possible. Haskins \& Wrigley de Basanta (2008) reported that, spore-to-spore culture had been achieved for approximately $10 \%$ of all known species of myxomycetes. Members of the Physarales are the most common species in culture and are assumed to be the easiest to cultivate under laboratory conditions (Haskins \& Wrigley de Basanta 2008). It has been known for a long time that species of myxomycetes display variation in germination when laboratory conditions are altered to include differences in abiotic factors such as $\mathrm{pH}$ associated with natural extracts in culture media (Smart 1937).

In light of the differences noted in the environmental conditions under which particular species are found in nature
(Stephenson et al. 2008), it may be that this ecological variation would translate to laboratory conditions as well.

Little data is available regarding intraspecific variation in cultivation success. Clark et al. (2002) obtained only 13 viable cultures that would go spore-to-spore for the myxomycete Arcyria cinerea from 55 field collections of the species. It is assumed that unequal cultivation success is a common occurrence, although little such information is found in the literature. The results of recent intraspecific molecular studies using DNA sequencing suggest that significant withinspecies variation is found at the molecular level (Winsett \& Stephenson 2008, 2011). How this relates to the recognized phenotypic variation in cosmopolitan species such as Didymium squamulosum is unknown.

Didymium squamulosum (Alb. \& Schwein.) Fr. (Fig. 1) is a common morphological species found worldwide (Martin \& Alexopoulos 1969). Myxomycetes 
can produce two vegetative forms, the amoeboflagellate, which multiply by mitosis and can coalesce to form a multinucleate plasmodium. Classification and species concepts are based upon the development of fruiting bodies from plasmodia in nature or laboratory culture. Complex reproductive strategies including heterothallic and nonheterothallic forms are known to occur in myxomycetes. D. squamulosum is known to include both types, and is thus considered to be a species complex of sibling biological species (El Hage et al. 2000). Significant morphological variation in $D$. squamulosum is recognized within the taxonomic description though it is, as yet, unclear how such variation is related to the known species complex (Martin \& Alexopoulos 1969, El Hage et al. 2000, Clark \& Haskins 2010, 2011).
In order to examine the the phenotypic variation of $D$. squamulosum under controlled laboratory conditions, and thus separate from any effect of variation in ecological condition, an attempt was made to cultivate 96 isolates of D. squamulosum from spore-to-spore, using standard culturing methods (e.g. Clark 1995, Haskins \& Wrigley de Basanta 2008, Haskins, personal communication; Wrigley de Basanta, personal communication). The specimens cultured originated from moist chamber cultures or field collections and represented a diversity of geographic localities, habitats and ages (Table 1). All attempts to culture these specimens were carried out during the summer and autumn of 2010. An initial method for germination and culture was attempted, followed by adaptations to the method in an effort to promote germination in those isolates that did not germinate during the first attempt.
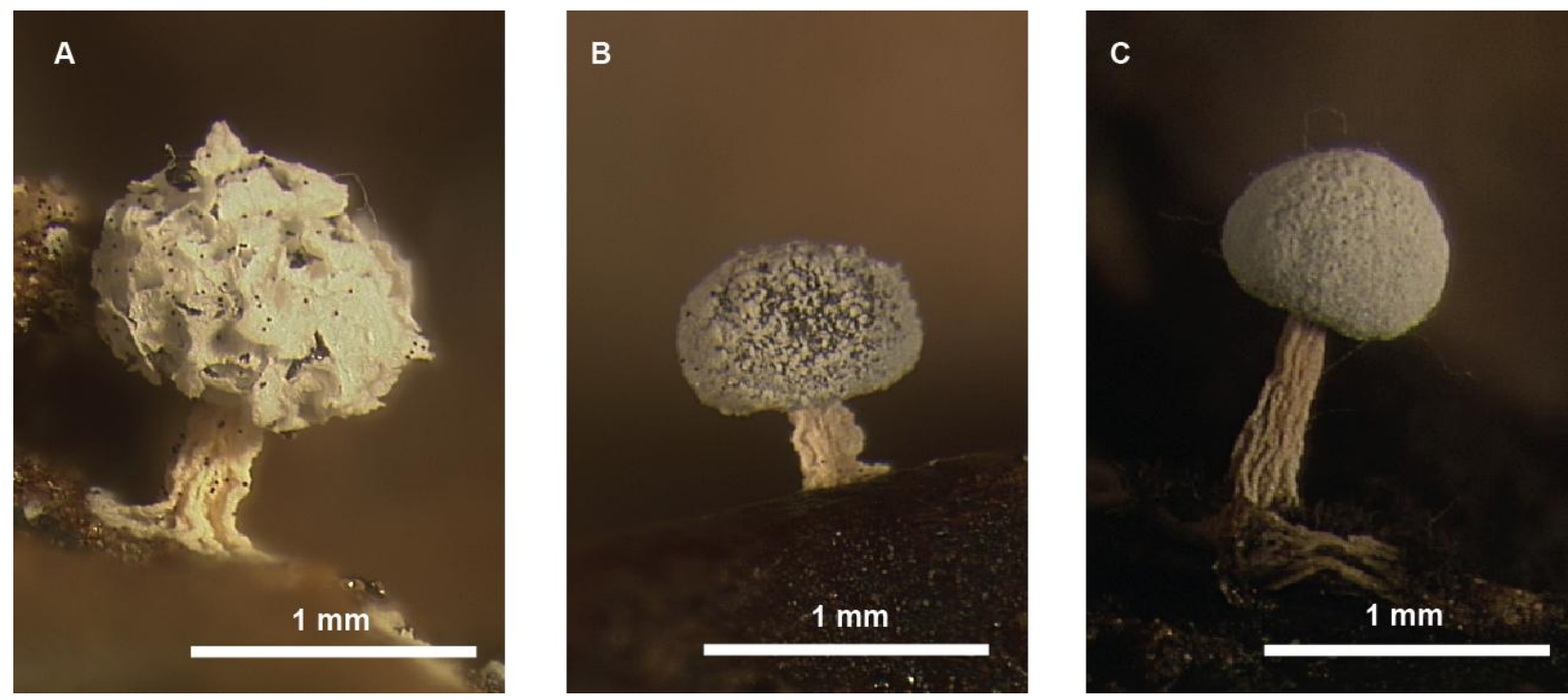

Fig 1 (A-C) - Three common morphological forms of Didymium squamulosum. Image A shows the typical flaky aspect to the peridium. Stalk variation common to the species is also shown, with images $\mathrm{A}$ and $\mathrm{B}$ showing the short stout stalk and image $\mathrm{C}$ indicating the longer stalk possible in this species. All three images show the fluted stalk typical of D. squamulosum. The stalk is commonly found with lime deposits present, most notably apparent in image A.

\section{Methods}

Spores were removed from 94
herbarium specimens of Didymium
squamulosum (Table 1). The specimens,
collected between 1998 and 2006, were housed
in the University of Arkansas myxomycete
herbarium (UARKM). Initially, spore
germination was attempted by placing most of
the spores from a single sporangium on sterile

$1.5 \%$ water agar plates (15 g Bacto agar, $1 \mathrm{~L}$ distilled water) with a surface film of sterile deionized water. Germination plates were stored at room temperature in indirect light. When germination occurred and strong populations of amoebae were found, a block of agar with amoebae present was placed upside down on the surface of sterile half-strength cornmeal agar plates $(\mathrm{CM} / 2)(8.5 \mathrm{~g}$ Bacto corn 
meal agar, $12.5 \mathrm{~g}$ Bacto agar, $1 \mathrm{~L}$ distilled water). Escherichia coli cells from liquid culture were streaked onto the plate to serve as food organisms. Following plasmodium formation, an agar block of plasmodium was transferred to a clean $\mathrm{CM} / 2$ plate, and crushed sterile oat flakes were added. Plasmodia were allowed to grow and fruit in natural light. Using these methods, spores from 31 specimens of $D$. squamulosum germinated.

An additional four specimens germinated following the use of an adaptation of the general method in which approximately $0.5 \mathrm{~mL}$ of a "natural substrate solution" was added to the plate of water agar on which spores were sown in mass. This solution was prepared by soaking a small piece of substrate material (approximately $1 \mathrm{~g}$ ) in $10 \mathrm{~mL}$ of sterile deionized water. The substrate material was taken from the herbarium specimen that served as the original source of the spores used for culturing. This technique was developed as a result of collaboration and discussions with Ms. Diana Wrigley de Basanta (see Wrigley de Basanta et al. 2010) and is based on the assumption that the mélange of biotic and abiotic factors present on this substrate favored fruiting, and likely germination, for the original isolate that fruited in nature or in a moist chamber culture.

For the cultures in which fruiting body production was observed, specimens were prepared for herbarium storage. The agar block substrate along with the fruiting bodies was cut out and glued to acid-free cardstock paper trays cut to fit into cardboard pill boxes. The specimens were accessioned with the same accession number as the original specimen and deposited in UARKM.

\section{Results}

Of the 94 spore-to-spore cultures attempted, less than $1 \%$ (nine) were successful. In $35(37.2 \%)$ cultures, spore germination occurred. Of these, only 13 formed plasmodia, nine of which resulted in fruiting body formation. No characteristic of the original herbarium specimen was common to all of the successful spore-to-spore cultures. Though most spores that successfully germinated in culture came from moist chamber, successful cultures were also started with spores from collections originating from field collections, aerial litter and ground litter, and from different geographic localities. Of the collections that only germinated in culture, variation in fruiting body source, substrate and locality was found. The only commonality that can be inferred relates to the age of the specimen.

No specimens that germinated were older than 6 years at time of agar culture. The oldest specimen that germinated was collected from a moist chamber culture in 2004, and the most recent were collected in October 2009 (less than one year since collection at the time the work was done). However, age of the collection could not predict whether germination would occur. Of the most recently collected specimens (2009), only four of 15 specimens germinated on agar.

\section{Discussion}

The absence of a pattern in the germination and growth success in spore-tospore culture of a single species of myxomycete suggests the existence of intraspecific variation that reflects varying responses to ecological conditions and requirements to complete the life cycle. Didymium squamulosum is a cosmopolitan morphological species found in a number of habitats and on various substrates. The variation in germination and growth described herein can be added to the significant morphological variation that is also recognized within this species (El Hage et al. 2000, Winsett, unpublished data). There is not yet any mechanism describing phenotypic plasticity in this or any other species of myxomycete, nor is there a complete understanding of intraspecific molecular groups or types. Recent data suggest that intraspecific groupings do exist within species, but these have not been connected to morphological variation (El Hage et al. 2000, Winsett \& Stephenson 2008, 2011, Winsett, unpublished data).

While no clear pattern could be detected regarding cultivation success, it must be noted that most of the successful isolates were obtained from moist chamber culture. Seven of the nine isolates that successfully went spore-to-spore in culture and nine of the 13 isolates that produced plasmodia originated 
Table 1 Collection data and culture results for each specimen included in the study. Accession numbers marked with an asterisk (*) are 14 collections that did not germinate using initial methods and were further studied using a mélange solution to promote germination.

\begin{tabular}{|c|c|c|c|c|c|c|c|}
\hline $\begin{array}{l}\text { Accession } \\
\text { number }\end{array}$ & $\begin{array}{l}\text { Geographic } \\
\text { origin }\end{array}$ & Substrate & $\begin{array}{l}\text { From } \\
\text { moist } \\
\text { chamber } \\
\text { (MC) } \\
\text { or field } \\
\text { collected } \\
\text { (FC) }\end{array}$ & $\begin{array}{l}\text { Year } \\
\text { collected }\end{array}$ & Germination & $\begin{array}{l}\text { Plasmodium } \\
\text { formation }\end{array}$ & $\begin{array}{l}\text { Fruiting } \\
\text { body } \\
\text { formation }\end{array}$ \\
\hline 24034 & $\begin{array}{l}\text { Hawaii, } \\
\text { USA }\end{array}$ & $\begin{array}{l}\text { Aerial } \\
\text { litter }\end{array}$ & $\mathrm{MC}$ & 2004 & $X$ & & \\
\hline 26253 & $\begin{array}{l}\text { North } \\
\text { Dakota, } \\
\text { USA }\end{array}$ & $\begin{array}{l}\text { Aerial } \\
\text { litter }\end{array}$ & $\mathrm{FC}$ & 2006 & $X$ & $\mathrm{X}$ & \\
\hline 26282 & $\begin{array}{l}\text { North } \\
\text { Dakota, } \\
\text { USA }\end{array}$ & $\begin{array}{l}\text { Aerial } \\
\text { litter }\end{array}$ & $\mathrm{FC}$ & 2006 & $X$ & $X$ & $\mathrm{X}$ \\
\hline 26283 & $\begin{array}{l}\text { Colorado, } \\
\text { USA }\end{array}$ & $\begin{array}{l}\text { Aerial } \\
\text { litter }\end{array}$ & $\mathrm{FC}$ & 2006 & $X$ & $\mathrm{X}$ & \\
\hline 26293 & $\begin{array}{l}\text { Kansas, } \\
\text { USA }\end{array}$ & $\begin{array}{l}\text { Aerial } \\
\text { litter }\end{array}$ & $\mathrm{FC}$ & 2006 & $X$ & & \\
\hline 26301 & $\begin{array}{l}\text { Oklahoma, } \\
\text { USA }\end{array}$ & $\begin{array}{l}\text { Aerial } \\
\text { litter }\end{array}$ & $\mathrm{FC}$ & 2006 & $X$ & & \\
\hline 26537 & $\begin{array}{l}\text { North } \\
\text { Dakota, } \\
\text { USA }\end{array}$ & $\begin{array}{l}\text { Aerial } \\
\text { litter }\end{array}$ & $\mathrm{FC}$ & 2006 & $\mathrm{X}$ & & \\
\hline 26573 & $\begin{array}{l}\text { North } \\
\text { Dakota, } \\
\text { USA }\end{array}$ & $\begin{array}{l}\text { Aerial } \\
\text { litter }\end{array}$ & $\mathrm{FC}$ & 2006 & $X$ & $\mathrm{X}$ & $\mathrm{X}$ \\
\hline 31858 & Guatemala & $\begin{array}{l}\text { Aerial } \\
\text { litter }\end{array}$ & $\mathrm{MC}$ & 2006 & $\mathrm{X}$ & & \\
\hline 31885 & Guatemala & $\begin{array}{l}\text { Aerial } \\
\text { litter }\end{array}$ & $\mathrm{MC}$ & 2007 & $X$ & & \\
\hline 31886 & Guatemala & $\begin{array}{l}\text { Aerial } \\
\text { litter }\end{array}$ & $\mathrm{MC}$ & 2007 & $X$ & & \\
\hline 31890 & Guatemala & $\begin{array}{l}\text { Aerial } \\
\text { litter }\end{array}$ & $\mathrm{MC}$ & 2007 & $X$ & & \\
\hline 31905 & Guatemala & $\begin{array}{l}\text { Ground } \\
\text { litter }\end{array}$ & $\mathrm{MC}$ & 2007 & $X$ & & \\
\hline 31907 & Guatemala & $\begin{array}{l}\text { Aerial } \\
\text { litter }\end{array}$ & $\mathrm{MC}$ & 2007 & $X$ & & \\
\hline 31910 & Guatemala & $\begin{array}{l}\text { Aerial } \\
\text { litter }\end{array}$ & $\mathrm{MC}$ & 2007 & $X$ & & \\
\hline 31922 & Guatemala & $\begin{array}{l}\text { Aerial } \\
\text { litter }\end{array}$ & $\mathrm{MC}$ & 2007 & $X$ & & \\
\hline 34688 & Guatemala & $\begin{array}{l}\text { Aerial } \\
\text { litter }\end{array}$ & $\mathrm{MC}$ & 2008 & $X$ & $X$ & $X$ \\
\hline 34689 & Guatemala & $\begin{array}{l}\text { Aerial } \\
\text { litter }\end{array}$ & $\mathrm{MC}$ & 2007 & $X$ & $X$ & \\
\hline 34691 & Guatemala & $\begin{array}{l}\text { Aerial } \\
\text { litter }\end{array}$ & $\mathrm{MC}$ & 2007 & $X$ & $\mathrm{X}$ & X \\
\hline
\end{tabular}


Table 1 continued. Collection data and culture results for each specimen included in the study. Accession numbers marked with an asterisk $(*)$ are 14 collections that did not germinate using initial methods and were further studied using a mélange solution to promote germination.

\begin{tabular}{|c|c|c|c|c|c|c|c|}
\hline $\begin{array}{l}\text { Accession } \\
\text { number }\end{array}$ & $\begin{array}{l}\text { Geographic } \\
\text { origin }\end{array}$ & Substrate & 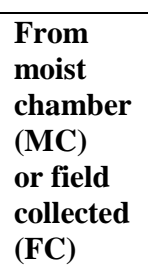 & $\begin{array}{l}\text { Year } \\
\text { collected }\end{array}$ & Germination & $\begin{array}{l}\text { Plasmodium } \\
\text { formation }\end{array}$ & $\begin{array}{l}\text { Fruiting } \\
\text { body } \\
\text { formation }\end{array}$ \\
\hline 34692 & Guatemala & $\begin{array}{l}\text { Aerial } \\
\text { litter }\end{array}$ & $\mathrm{MC}$ & 2007 & $X$ & & \\
\hline 34697 & Guatemala & $\begin{array}{l}\text { Aerial } \\
\text { litter }\end{array}$ & $\mathrm{MC}$ & 2007 & $X$ & $X$ & $X$ \\
\hline 34711 & Guatemala & $\begin{array}{l}\text { Aerial } \\
\text { litter }\end{array}$ & $\mathrm{MC}$ & 2007 & $X$ & & \\
\hline 34729 & Guatemala & $\begin{array}{l}\text { Aerial } \\
\text { litter }\end{array}$ & $\mathrm{MC}$ & 2007 & $\mathrm{X}$ & $X$ & $X$ \\
\hline 34900 & $\begin{array}{l}\text { South } \\
\text { Africa }\end{array}$ & $\begin{array}{l}\text { Ground } \\
\text { litter }\end{array}$ & $\mathrm{MC}$ & 2006 & $X$ & & \\
\hline 35278 & $\begin{array}{l}\text { South } \\
\text { Africa }\end{array}$ & $\begin{array}{l}\text { Ground } \\
\text { litter }\end{array}$ & $\mathrm{MC}$ & 2006 & $X$ & $X$ & \\
\hline 38107 & $\begin{array}{l}\text { South } \\
\text { Africa }\end{array}$ & $\begin{array}{l}\text { Ground } \\
\text { litter }\end{array}$ & $\mathrm{MC}$ & 2006 & $X$ & & \\
\hline 38162 & $\begin{array}{l}\text { South } \\
\text { Africa }\end{array}$ & $\begin{array}{l}\text { Aerial } \\
\text { litter }\end{array}$ & $\mathrm{MC}$ & 2006 & $X$ & & \\
\hline 38173 & $\begin{array}{l}\text { South } \\
\text { Africa }\end{array}$ & $\begin{array}{l}\text { Coarse } \\
\text { woody } \\
\text { debris }\end{array}$ & $\mathrm{MC}$ & 2006 & $X$ & & \\
\hline 38855 & $\begin{array}{l}\text { South } \\
\text { Africa }\end{array}$ & $\begin{array}{l}\text { Aerial } \\
\text { litter }\end{array}$ & $\mathrm{MC}$ & 2006 & $X$ & $X$ & $X$ \\
\hline 38860 & $\begin{array}{l}\text { South } \\
\text { Africa }\end{array}$ & $\begin{array}{l}\text { Aerial } \\
\text { litter }\end{array}$ & $\mathrm{MC}$ & 2006 & $X$ & $X$ & $X$ \\
\hline 38864 & $\begin{array}{l}\text { South } \\
\text { Africa }\end{array}$ & $\begin{array}{l}\text { Ground } \\
\text { litter }\end{array}$ & $\mathrm{MC}$ & 2006 & $X$ & $\mathrm{X}$ & $X$ \\
\hline$* 43763$ & $\begin{array}{l}\text { New } \\
\text { Zealand }\end{array}$ & $\begin{array}{l}\text { Palm } \\
\text { frond }\end{array}$ & $\mathrm{FC}$ & 2009 & $X$ & & \\
\hline *43785 & $\begin{array}{l}\text { New } \\
\text { Zealand }\end{array}$ & $\begin{array}{l}\text { Palm } \\
\text { frond }\end{array}$ & $\mathrm{FC}$ & 2009 & $X$ & & \\
\hline$* 43786$ & $\begin{array}{l}\text { New } \\
\text { Zealand }\end{array}$ & $\begin{array}{l}\text { Palm } \\
\text { frond }\end{array}$ & $\mathrm{FC}$ & 2009 & $X$ & & \\
\hline *43790 & $\begin{array}{l}\text { New } \\
\text { Zealand }\end{array}$ & $\begin{array}{l}\text { Palm } \\
\text { frond }\end{array}$ & $\mathrm{FC}$ & 2009 & $X$ & & \\
\hline 9727 & $\begin{array}{l}\text { New } \\
\text { Zealand }\end{array}$ & $\begin{array}{l}\text { Palm } \\
\text { frond }\end{array}$ & $\mathrm{FC}$ & 1998 & & & \\
\hline 24034 & $\begin{array}{l}\text { Hawaii, } \\
\text { USA }\end{array}$ & $\begin{array}{l}\text { Aerial } \\
\text { litter }\end{array}$ & $\mathrm{FC}$ & 2004 & & & \\
\hline 26038 & $\begin{array}{l}\text { North } \\
\text { Dakota, } \\
\text { USA }\end{array}$ & $\begin{array}{l}\text { Aerial } \\
\text { litter }\end{array}$ & $\mathrm{FC}$ & 2006 & & & \\
\hline 26039 & $\begin{array}{l}\text { North } \\
\text { Dakota, } \\
\text { USA }\end{array}$ & $\begin{array}{l}\text { Aerial } \\
\text { litter }\end{array}$ & $\mathrm{FC}$ & 2006 & & & \\
\hline
\end{tabular}


Table 1 continued. Collection data and culture results for each specimen included in the study. Accession numbers marked with an asterisk (*) are 14 collections that did not germinate using initial methods and were further studied using a mélange solution to promote germination.

\begin{tabular}{|c|c|c|c|c|c|c|c|}
\hline $\begin{array}{l}\text { Accession } \\
\text { number }\end{array}$ & $\begin{array}{l}\text { Geographic } \\
\text { origin }\end{array}$ & Substrate & $\begin{array}{l}\text { From } \\
\text { moist } \\
\text { chamber } \\
\text { (MC) } \\
\text { or field } \\
\text { collected } \\
\text { (FC) }\end{array}$ & $\begin{array}{l}\text { Year } \\
\text { collected }\end{array}$ & Germination & $\begin{array}{l}\text { Plasmodium } \\
\text { formation }\end{array}$ & $\begin{array}{l}\text { Fruiting } \\
\text { body } \\
\text { formation }\end{array}$ \\
\hline 26260 & $\begin{array}{l}\text { Oklahoma, } \\
\text { USA }\end{array}$ & $\begin{array}{l}\text { Aerial } \\
\text { litter }\end{array}$ & $\mathrm{FC}$ & 2006 & & & \\
\hline 26263 & $\begin{array}{l}\text { North } \\
\text { Dakota, } \\
\text { USA }\end{array}$ & $\begin{array}{l}\text { Aerial } \\
\text { litter }\end{array}$ & $\mathrm{FC}$ & 2006 & & & \\
\hline 26266 & $\begin{array}{l}\text { Wyoming, } \\
\text { USA }\end{array}$ & $\begin{array}{l}\text { Aerial } \\
\text { litter }\end{array}$ & $\mathrm{FC}$ & 2006 & & & \\
\hline 26285 & $\begin{array}{l}\text { Kansas, } \\
\text { USA }\end{array}$ & $\begin{array}{l}\text { Ground } \\
\text { litter }\end{array}$ & $\mathrm{FC}$ & 2006 & & & \\
\hline 26309 & $\begin{array}{l}\text { Kansas, } \\
\text { USA }\end{array}$ & $\begin{array}{l}\text { Aerial } \\
\text { litter }\end{array}$ & $\mathrm{FC}$ & 2006 & & & \\
\hline 26558 & $\begin{array}{l}\text { New } \\
\text { Mexico, } \\
\text { USA }\end{array}$ & $\begin{array}{l}\text { Aerial } \\
\text { litter }\end{array}$ & $\mathrm{FC}$ & 2006 & & & \\
\hline 26592 & $\begin{array}{l}\text { Colorado, } \\
\text { USA }\end{array}$ & $\begin{array}{l}\text { Aerial } \\
\text { litter }\end{array}$ & $\mathrm{FC}$ & 2006 & & & \\
\hline 26607 & $\begin{array}{l}\text { North } \\
\text { Dakota, } \\
\text { USA }\end{array}$ & $\begin{array}{l}\text { Aerial } \\
\text { litter }\end{array}$ & $\mathrm{FC}$ & 2006 & & & \\
\hline 26644 & $\begin{array}{l}\text { Kansas, } \\
\text { USA }\end{array}$ & $\begin{array}{l}\text { Aerial } \\
\text { litter }\end{array}$ & $\mathrm{FC}$ & 2006 & & & \\
\hline 26689 & $\begin{array}{l}\text { Kansas, } \\
\text { USA }\end{array}$ & $\begin{array}{l}\text { Aerial } \\
\text { litter }\end{array}$ & $\mathrm{FC}$ & 2006 & & & \\
\hline 26848 & $\begin{array}{l}\text { New } \\
\text { Zealand }\end{array}$ & $\begin{array}{l}\text { Palm } \\
\text { petiole }\end{array}$ & $\mathrm{FC}$ & 2002 & & & \\
\hline 27869 & $\begin{array}{l}\text { New } \\
\text { Zealand }\end{array}$ & $\begin{array}{l}\text { Palm } \\
\text { frond }\end{array}$ & $\mathrm{FC}$ & 1998 & & & \\
\hline 28032 & $\begin{array}{l}\text { New } \\
\text { Zealand }\end{array}$ & $\begin{array}{l}\text { Palm } \\
\text { frond }\end{array}$ & $\mathrm{FC}$ & 1998 & & & \\
\hline 28035 & $\begin{array}{l}\text { New } \\
\text { Zealand }\end{array}$ & $\begin{array}{l}\text { Palm } \\
\text { frond }\end{array}$ & $\mathrm{FC}$ & 1998 & & & \\
\hline 28046 & $\begin{array}{l}\text { New } \\
\text { Zealand }\end{array}$ & $\begin{array}{l}\text { Palm } \\
\text { frond }\end{array}$ & $\mathrm{FC}$ & 1998 & & & \\
\hline 28062 & $\begin{array}{l}\text { New } \\
\text { Zealand }\end{array}$ & $\begin{array}{l}\text { Palm } \\
\text { frond }\end{array}$ & $\mathrm{FC}$ & 1998 & & & \\
\hline 28074 & $\begin{array}{l}\text { New } \\
\text { Zealand }\end{array}$ & $\begin{array}{l}\text { Palm } \\
\text { frond }\end{array}$ & $\mathrm{FC}$ & 1998 & & & \\
\hline 28079 & $\begin{array}{l}\text { New } \\
\text { Zealand }\end{array}$ & $\begin{array}{l}\text { Palm } \\
\text { frond }\end{array}$ & $\mathrm{FC}$ & 1998 & & & \\
\hline 28100 & $\begin{array}{l}\text { New } \\
\text { Zealand }\end{array}$ & $\begin{array}{l}\text { Palm } \\
\text { frond }\end{array}$ & $\mathrm{FC}$ & 1998 & & & \\
\hline
\end{tabular}


Table 1 continued. Collection data and culture results for each specimen included in the study. Accession numbers marked with an asterisk (*) are 14 collections that did not germinate using initial methods and were further studied using a mélange solution to promote germination.

\begin{tabular}{|c|c|c|c|c|c|c|c|}
\hline $\begin{array}{l}\text { Accession } \\
\text { number }\end{array}$ & $\begin{array}{l}\text { Geographic } \\
\text { origin }\end{array}$ & Substrate & 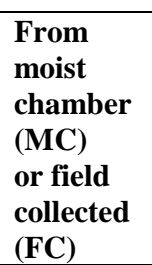 & $\begin{array}{l}\text { Year } \\
\text { collected }\end{array}$ & Germination & $\begin{array}{l}\text { Plasmodium } \\
\text { formation }\end{array}$ & $\begin{array}{l}\text { Fruiting } \\
\text { body } \\
\text { formation }\end{array}$ \\
\hline 28105 & $\begin{array}{l}\text { New } \\
\text { Zealand }\end{array}$ & $\begin{array}{l}\text { Palm } \\
\text { frond }\end{array}$ & $\mathrm{FC}$ & 1998 & & & \\
\hline 28126 & $\begin{array}{l}\text { New } \\
\text { Zealand }\end{array}$ & $\begin{array}{l}\text { Aerial } \\
\text { litter }\end{array}$ & $\mathrm{MC}$ & 1998 & & & \\
\hline 28151 & $\begin{array}{l}\text { New } \\
\text { Zealand }\end{array}$ & $\begin{array}{l}\text { Palm } \\
\text { frond }\end{array}$ & $\mathrm{FC}$ & 1998 & & & \\
\hline 28239 & $\begin{array}{l}\text { New } \\
\text { Zealand }\end{array}$ & $\begin{array}{l}\text { Palm } \\
\text { frond }\end{array}$ & $\mathrm{FC}$ & 1998 & & & \\
\hline 31864 & Guatemala & $\begin{array}{l}\text { Aerial } \\
\text { litter }\end{array}$ & $\mathrm{MC}$ & 2007 & & & \\
\hline 31884 & Guatemala & $\begin{array}{l}\text { Ground } \\
\text { litter }\end{array}$ & $\mathrm{MC}$ & 2007 & & & \\
\hline 31898 & Guatemala & $\begin{array}{l}\text { Ground } \\
\text { litter }\end{array}$ & $\mathrm{MC}$ & 2007 & & & \\
\hline 31903 & Guatemala & $\begin{array}{l}\text { Coarse } \\
\text { woody } \\
\text { debris }\end{array}$ & $\mathrm{MC}$ & 2007 & & & \\
\hline 34581 & $\begin{array}{l}\text { South } \\
\text { Africa }\end{array}$ & $\begin{array}{l}\text { Ground } \\
\text { litter }\end{array}$ & $\mathrm{MC}$ & 2006 & & & \\
\hline 34583 & $\begin{array}{l}\text { South } \\
\text { Africa }\end{array}$ & $\begin{array}{l}\text { Ground } \\
\text { litter }\end{array}$ & $\mathrm{MC}$ & 2006 & & & \\
\hline 34661 & Guatemala & $\begin{array}{l}\text { Aerial } \\
\text { litter }\end{array}$ & $\mathrm{MC}$ & 2007 & & & \\
\hline 34693 & Guatemala & $\begin{array}{l}\text { Aerial } \\
\text { litter }\end{array}$ & $\mathrm{MC}$ & 2007 & & & \\
\hline 34857 & $\begin{array}{l}\text { South } \\
\text { Africa }\end{array}$ & $\begin{array}{l}\text { Aerial } \\
\text { litter }\end{array}$ & $\mathrm{MC}$ & 2006 & & & \\
\hline *43742 & $\begin{array}{l}\text { New } \\
\text { Zealand }\end{array}$ & $\begin{array}{l}\text { Palm } \\
\text { frond }\end{array}$ & $\mathrm{FC}$ & 2009 & & & \\
\hline$* 43745$ & $\begin{array}{l}\text { New } \\
\text { Zealand }\end{array}$ & $\begin{array}{l}\text { Palm } \\
\text { frond }\end{array}$ & $\mathrm{FC}$ & 2009 & & & \\
\hline *43746 & $\begin{array}{l}\text { New } \\
\text { Zealand }\end{array}$ & $\begin{array}{l}\text { Palm } \\
\text { frond }\end{array}$ & $\mathrm{FC}$ & 2009 & & & \\
\hline$* 43761$ & $\begin{array}{l}\text { New } \\
\text { Zealand }\end{array}$ & $\begin{array}{l}\text { Palm } \\
\text { frond }\end{array}$ & $\mathrm{FC}$ & 2009 & & & \\
\hline$* 43762$ & $\begin{array}{l}\text { New } \\
\text { Zealand }\end{array}$ & $\begin{array}{l}\text { Palm } \\
\text { frond }\end{array}$ & $\mathrm{FC}$ & 2009 & & & \\
\hline$* 43773$ & $\begin{array}{l}\text { New } \\
\text { Zealand }\end{array}$ & $\begin{array}{l}\text { Palm } \\
\text { frond }\end{array}$ & $\mathrm{FC}$ & 2009 & & & \\
\hline *43777 & $\begin{array}{l}\text { New } \\
\text { Zealand }\end{array}$ & $\begin{array}{l}\text { Palm } \\
\text { frond }\end{array}$ & $\mathrm{FC}$ & 2009 & & & \\
\hline$* 43787$ & $\begin{array}{l}\text { New } \\
\text { Zealand }\end{array}$ & $\begin{array}{l}\text { Palm } \\
\text { frond }\end{array}$ & $\mathrm{FC}$ & 2009 & & & \\
\hline
\end{tabular}


Table 1 continued. Collection data and culture results for each specimen included in the study. Accession numbers marked with an asterisk (*) are 14 collections that did not germinate using initial methods and were further studied using a mélange solution to promote germination.

\begin{tabular}{|c|c|c|c|c|c|c|c|}
\hline $\begin{array}{l}\text { Accession } \\
\text { number }\end{array}$ & $\begin{array}{l}\text { Geographic } \\
\text { origin }\end{array}$ & Substrate & 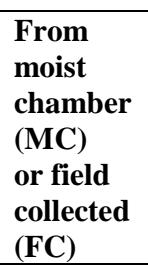 & $\begin{array}{l}\text { Year } \\
\text { collected }\end{array}$ & Germination & $\begin{array}{l}\text { Plasmodium } \\
\text { formation }\end{array}$ & $\begin{array}{l}\text { Fruiting } \\
\text { body } \\
\text { formation }\end{array}$ \\
\hline$* 43791$ & $\begin{array}{l}\text { New } \\
\text { Zealand }\end{array}$ & $\begin{array}{l}\text { Palm } \\
\text { frond }\end{array}$ & $\mathrm{FC}$ & 2009 & & & \\
\hline *43792 & $\begin{array}{l}\text { New } \\
\text { Zealand }\end{array}$ & $\begin{array}{l}\text { Palm } \\
\text { frond }\end{array}$ & $\mathrm{FC}$ & 2009 & & & \\
\hline 44647 & $\begin{array}{l}\text { New } \\
\text { Zealand }\end{array}$ & $\begin{array}{l}\text { Ground } \\
\text { litter }\end{array}$ & $\mathrm{FC}$ & 2001 & & & \\
\hline 44674 & $\begin{array}{l}\text { New } \\
\text { Zealand }\end{array}$ & $\begin{array}{l}\text { Palm } \\
\text { petiole }\end{array}$ & $\mathrm{FC}$ & 2005 & & & \\
\hline 44690 & $\begin{array}{l}\text { New } \\
\text { Zealand }\end{array}$ & $\begin{array}{l}\text { Palm } \\
\text { petiole }\end{array}$ & $\mathrm{FC}$ & 2006 & & & \\
\hline 44973 & $\begin{array}{l}\text { New } \\
\text { Zealand }\end{array}$ & $\begin{array}{l}\text { Palm } \\
\text { petiole }\end{array}$ & $\mathrm{FC}$ & 2005 & & & \\
\hline 44974 & $\begin{array}{l}\text { New } \\
\text { Zealand }\end{array}$ & $\begin{array}{l}\text { Palm } \\
\text { petiole }\end{array}$ & $\mathrm{FC}$ & 2006 & & & \\
\hline 44975 & $\begin{array}{l}\text { New } \\
\text { Zealand }\end{array}$ & $\begin{array}{l}\text { Palm } \\
\text { petiole }\end{array}$ & $\mathrm{FC}$ & 2006 & & & \\
\hline 44976 & $\begin{array}{l}\text { New } \\
\text { Zealand }\end{array}$ & $\begin{array}{l}\text { Palm } \\
\text { petiole }\end{array}$ & $\mathrm{FC}$ & 2006 & & & \\
\hline 44977 & $\begin{array}{l}\text { New } \\
\text { Zealand }\end{array}$ & $\begin{array}{l}\text { Palm } \\
\text { frond }\end{array}$ & $\mathrm{FC}$ & 2009 & & & \\
\hline 44979 & $\begin{array}{l}\text { New } \\
\text { Zealand }\end{array}$ & $\begin{array}{l}\text { Palm } \\
\text { frond }\end{array}$ & $\mathrm{FC}$ & 2005 & & & \\
\hline 44981 & Costa Rica & $\begin{array}{l}\text { Flower } \\
\text { spike }\end{array}$ & $\mathrm{FC}$ & 1999 & & & \\
\hline CSAK013 & $\begin{array}{l}\text { New } \\
\text { Zealand }\end{array}$ & $\begin{array}{l}\text { Palm } \\
\text { petiole }\end{array}$ & $\mathrm{FC}$ & 2001 & & & \\
\hline CSAK049 & $\begin{array}{l}\text { New } \\
\text { Zealand }\end{array}$ & $\begin{array}{l}\text { Palm } \\
\text { petiole }\end{array}$ & $\mathrm{FC}$ & 2002 & & & \\
\hline OTA58428 & $\begin{array}{l}\text { New } \\
\text { Zealand }\end{array}$ & $\begin{array}{l}\text { Ground } \\
\text { litter }\end{array}$ & $\mathrm{FC}$ & 2004 & & & \\
\hline
\end{tabular}

from fruiting bodies that developed in moist chamber culture of substrate material. Perhaps growth in moist chamber culture provides opportunity for full maturation of the sporocarp. However, instances have been personally observed when spores of fruiting bodies in moist chamber culture developed abnormally suggesting care must be taken to allow fruiting bodies to fully mature in these conditions as well as examination of spores to determine if normal development occurred.
The technique of using a mélange solution from soaking substrate material was attempted for 14 isolates, all of which were collected at the same locality in October 2009 (Table 1). These isolates all fruited over the same few days with very similar morphology and identical sequences for a mitochondrial marker (Winsett, unpublished data). Because D. squamulosum is known to occur as nonheterothallic (presumably apomictic) strains (El Hage et al. 2000, Clark \& Stephenson 2003), it was assumed that these field collections could 
be different isolates of the same genetic strain. Furthermore, the assumption was made that these isolates would behave in a similar fashion in culture, with similar germination rates and growth success. Interestingly, despite their recent collection, none of the isolates germinated on water agar alone, and only four of the 16 isolates germinated using the substrate mélange technique. If these specimens were members of the same clone, it would be reasonable to assume they would behave similarly in culture, responding the same way to any set of culture conditions.

Along with the biological conclusions that can be drawn from these data, it should also be noted that the interpretation of data from laboratory cultivation, meant to investigate if phenotypic variation holds up in common culture conditions, could be made more difficult by the unequal success of germination within a particular species. This series of spore-to-spore culture attempts in $D$. squamulosum was undertaken to enhance an intraspecific molecular study of the same species. The expectation was to use the fruiting bodies formed in culture to determine if the extensive morphological variation seen in these herbarium specimens would be seen also when cultivated in common conditions. $D$. squamulosum is known to occur, perhaps commonly, as non-heterothallic strains (El Hage et al. 2000, Clark \& Stephenson 2003). Such genetic isolation would serve as a mechanism for the accumulation of phenotypic variation within morphological species. Understanding whether the source of morphological variation could be ecological or genetic using evidence from whether or not variation was sustained in common culture would be important for investigating myxomycete species. The value of the information that can be drawn from the laboratory cultivation of myxomycete isolates has been significantly reviewed (Clark \& Haskins 2010, 2011). Uneven germination rates and the inability to predict which specimens will be successful in spore-to-spore culture is a limitation in the study of the morphological species concept.

\section{Acknowledgements}

This project was supported by National Science Foundation Planetary Biodiversity Inventory (PBI) grant (DEB-0316284; PI Steven L. Stephenson) and NSF Partnerships for Enhancing Expertise in Taxonomy (PEET) grant (DEB-0329102; PI Frederick W. Spiegel) at the University of Arkansas. George Ndiritu, Carlos Rojas, Adam W. Rollins, Clive Shirley and Steven L. Stephenson are gratefully acknowledged for providing many of the specimens used in this study.

\section{References}

Clark JD. 1995 - Plasmodial incompatibility in the myxomycete Didymium squamulosum. Mycologia 95, 24-26.

Clark JD, Haskins EF. 2010 - Reproductive systems in the myxomycetes: a review. Mycosphere 1, 337-353.

Clark JD, Haskins EF. 2011 - Principles and protocols for genetical study of myxomycete reproductive systems and plasmodial coalescence. Mycosphere 2, 487-496.

Clark JD, Stephenson, SL. 2003 Biosystematics of the myxomycetes Didymium squamulosum, Physarum compressum and Physarum melleum: additional isolates. Mycotaxon 85, 85-89.

Clark JD, Schnittler M, Stephenson SL. 2002 Biosystematics of the myxomycete Arcyria cinerea. Mycotaxon 82, 343-346.

El Hage, N, Little, C Clark, JD, Stephenson SL. 2000 - Biosystematics of the Didymium squamulosum complex. Mycologia 92, 54-64.

Haskins EF, Wrigley de Basanta D. 2008 Methods of agar culture of myxomycetes: an overview. Revista Mexicana de Micología 27, 1-7.

Lister A. 1901 - On the cultivation of Mycetozoa from spores. Journal of Botany 39, 5-8.

Martin GW, Alexopoulos CJ. 1969 - The Myxomycetes. University of Iowa Press, Iowa City.

Smart RF. 1937 - Influence of certain external factors on spore germination in the myxomycetes. American Journal of Botany 24, 145-159. 
Stephenson SL, Schnittler, M, Novozhilov, YK. 2008 - Myxomycete diversity and distribution from the fossil record to the present. Biodiversity and Conservation 17, 285-301.

Winsett KE, Stephenson SL. 2008 - Using ITS sequences to assess intraspecific genetic relationships among geographically separated collections of the myxomycete Didymium squamulosum. Revista Mexicana de Micología 27, 59-65.
Winsett KE, Stephenson SL. 2011 - Global distribution and molecular diversity of Didymium difforme. Mycosphere 2, 135146.

Wrigley de Basanta D, Lado C, Estrada-Torres A. 2010 - Licea eremophila, a new myxomycete from arid areas of South America. Mycologia 102, 1185-1192. 